\title{
A Finite Element Approach to the Behavior of the ITZ
}

\author{
Han Ay Lie ${ }^{1, a}$ and Sri Tudjono ${ }^{2, b}$ \\ ${ }^{1}$ Department of Civil Engineering, Diponegoro University, Semarang, Indonesia \\ ${ }^{2}$ The Structural and Material Laboratory, Diponegoro University, Semarang, Indonesia \\ ahanaylie@indosat.net.id, ${ }^{b}$ tudjono@gmail.com
}

\begin{abstract}
Keywords: Interfacial Transition Zone, Finite Element Model, Linkage Element.
\end{abstract}
\begin{abstract}
The Interfacial Transition Zone (ITZ) between two basic materials having differentiations in mechanical properties has always been intriguing. The stiffness disparities between the two will result in a very distinctive area, the interface. Cement based components such as mortar and concrete consist of the cement paste and aggregates, with the ITZ at the perimeter. When compared to the cement paste, this ITZ has a higher porosity with a dissimilar crystal formation. The resulting area therefore becomes the weak link in concrete. A Finite Element Model (FEM) was developed to construct the load-displacement behavior of a single inclusion specimen and to study the crack propagation within the ITZ. The ITZ was modeled as a linkage element having a double spring, perpendicular and parallel to the ITZ surface. The individual stiffness behavior of these springs was obtained from laboratory-tested specimens. Non-linearity was generated by evaluating the principal stresses and strains at Gauss points, while the CEB-FIB 2010 code was used for the constitutive material behavior of the mortar. Iteration is conducted by the arc-length method developed by Riks-Wempners. The load-displacement curves resulting from the FEM were validated with laboratory tested specimens to compare its effectiveness and assess the correctness of the model.
\end{abstract}

\section{Introduction}

The Interfacial Transition Zone (ITZ). The Interfacial Transition Zone (ITZ) in concrete is the weak link within the material. This weakness is due to the low adhesive strength, originating from the high volume and size of voids in this area. Additionally, the large crystals, especially calcium hydroxides, have an orientation that promotes crack propagation along their weak bond plane, following the Van der Waals's path of forces.

Laboratory techniques and methods to obtain information on the ITZ are limited and only provide a small range of data on the subject. A list of the most recent testing methods were compiled and presented in the reference [1]. The construction of a Finite Element Model (FEM) was therefore the chosen approach to obtain a better understanding of the ITZ, its influence on the structure and the overall behavior of the composite material.

The majority of the FEM represents the bond in the ITZ by the smeared-crack method. This approach allows cracks to propagate along the ITZ surface. While the constitutive model for the bond relationship was highly simplified, this method has its weakness in that no bond failure will occur [2]. In this study, the FEM model was calibrated by the inverse modeling technique, laboratory specimens were constructed to function as calibrating tool for the FEM. The mortar matrix was assumed as a continuum element, and the ITZ itself was considered homogeneous; variations along the aggregate surface were not incorporated into the model.

The ITZ Finite Element Model. The ITZ was modeled as a linkage element consisting of two one-degree-of-freedom springs. The first spring was perpendicular to the aggregate surface, representing the ITZ in tension, and the second parallel, illustrating the shear response. In meshing, this ITZ was constructed by assigning two nodes having identical coordinates.

The behavior of the ITZ was expressed by the two spring constants, $\mathrm{k}_{\eta}$ in tension and $\mathrm{k}_{\xi}$ in shear. The behavior of these constants were conveyed in their load-displacement responses, obtained through uniquely developed laboratory tests $[1,3,4]$. The response of the ITZ under increasing load 
was converted to the local coordinate system $\eta-\xi$ to update the corresponding ITZ stiffness. The factors influencing the ITZ load-displacement behavior are among others: the roughness of the aggregate surface, the mechanical properties of the mortar and the aggregate, the water-cement ratio, secondary reactions in the ITZ, and the presence of bleeding. The tensile behavior was obtained by recording the response of an axially loaded cylindrical aggregate attached to a mortar surface. The shear response was recorded from a second testing procedure, where an axial compression load was applied to a specially prepared specimen, embedded in mortar.

The load-displacement response in tension was characterized by a polynomial to the second degree. The curve showed a distinctively non-linear behavior, even at very low loading levels. Failure occurred due to bond loss in the ITZ. The load-displacement relationship for the ITZ in shear had a bi-linear function, the first part representing the stiffness modulus as a contribution of adhesion and friction, and the second exclusively being the result of friction and fine aggregate interlocking (Fig. 1). The outcome of the finite element analysis confirmed that failure in the ITZ was initiated in the tension areas.

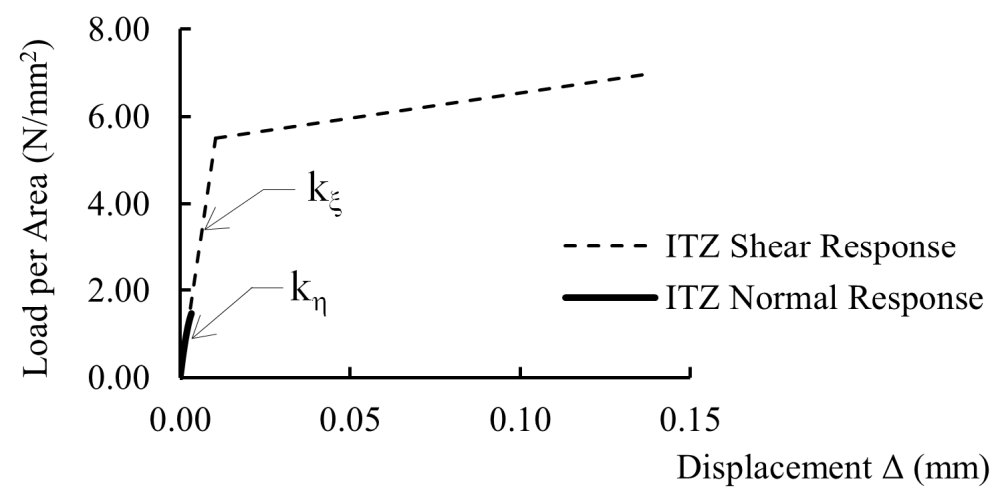

Fig. 1 Constitutive models for the ITZ behavior

\section{Finite Element Analysis}

Linkage Element Behavior. Since the relative displacement of the two adjacent nodes in the ITZ represented the behavior of the springs, these displacements were transformed from the global coordinate system $(X, Y)$ to the local coordinate system $(\eta, \xi)$. The local coordinate system was demarcated at the bisection line of the angle between the two elements of the ITZ.

Failure Criteria. The FEM consisted of the ITZ, the mortar matrix and the aggregate. The matrix was evaluated based on the principal stresses at the Gauss points. The failure criteria were distinguished either as crushing or fracturing of the mortar matrix, based on the Kupfer-Hilsdorf-Rusch's failure envelope. Fracture of a Gauss point under a certain loading increment reduced the stiffness of its element, and progressive loading lead to failure of one or more Gauss points up till collapse of the element as a whole [5].

Material Non-linearity, Isotropic and Anisotropic Behavior. The failure criteria were expressed in the hydrostatic system as $f(\xi, \rho, \theta)=0$. Tensile stresses were signed positive and compression negative. Further, Ottosen developed an algorithm to incorporate non-linearity by introducing the non-linearity index $\beta$, relating the actual most compressive principal stress to the stress at failure in compression [6]. At failure, the value of $\beta$ equals one. This model was formulated assuming that the principal axes of material anisotropy coincide with the principal stress directions. The tangent modulus in the anisotropic axes system was obtained from the corresponding stress-strain relationship, while the material stiffness matrix as proposed by Chen and Chen and Saleeb [7, 8] was chosen for the finite element algorithms. The Poisson ratio was considered a constant.

The compression-tension bi-axial stress combination was the most sensitive due to the shape of the failure envelope. Within the limits of the envelope, the assumption that the material behavior is isotropic was sustained, especially since stress and strain levels were very low. The initial Young's tangent stiffness modulus therefore gave a good representation of the actual behavior. 
When cracks started to propagate, and the bi-axial principal stresses exceeded the failure envelope boundaries, the anisotropic model was accessed. Based on research work done by Vecchio and Collins, the rotation angle for stresses closely approaches the rotation angle for strain [9]; hence, the principal strains were obtained based on the rotation angle of stresses and their corresponding strains.

Programming Language and Iteration Techniques. To obtain the coordinates of the element nodes and the ITZ, a mesh generator was used to create double nodes along this ITZ. The output of the mesh generator was called by the Visual Basic (Microsoft Visual Studio 2008) program to support the finite element analysis. Based on preliminary studies and the observation of the crack pattern of laboratory specimens, finer meshing was placed in the areas that were most vulnerable to high principal stresses. To accommodate the non-linearity of the model, the arch-length iteration technique was applied.

\section{Result and Discussion}

Evaluation and Validation of the FEM. The model was run for a mortar cube 100x100x50 mm in dimension, having a single cylindrical aggregate inclusion, $20.80 \mathrm{~mm}$ in diameter. The mechanical properties of the mortar, aggregate and the ITZ were obtained from laboratory-tested specimens and are listed in Table 1.

To evaluate the correctness of the ITZ algorithms, the response of the spring in the normal direction was tested for an arbitrarily load. Fig. 2 shows the response of the springs in the model. The gray dots represent the normal linkage element in tension, while the black dots represent compression. The outcome of the FEM program was confirmed by the visual observation of the laboratory tested specimens.

Table 1 Material Properties

\begin{tabular}{ccccc}
\hline \multirow{2}{*}{ Properties } & \multicolumn{3}{c}{ Material } & \multicolumn{2}{c}{ ITZ } \\
\cline { 2 - 5 } & mortar & aggregate & normal & shear \\
\hline$f_{c}^{\prime}[\mathrm{MPa}]$ & 27.3 & 169.9 & - & - \\
$E[\mathrm{GPa}]$ & 30.0 & 57.6 & - & - \\
Poisson's ratio v & 0.23 & 0.25 & - & - \\
$E_{0}\left[\mathrm{~N} / \mathrm{mm}^{2} / \mathrm{mm}\right]$ & - & - & 667.4 & 523.6 \\
$\varepsilon_{0}[\mathrm{~mm} / \mathrm{mm}]$ & - & - & 0.00033 & 0.00105 \\
A coefficient $\left(\mathrm{P}=\mathrm{A} \Delta^{2}+\mathrm{B}\right)$ & - & - & -67690 & - \\
\hline
\end{tabular}
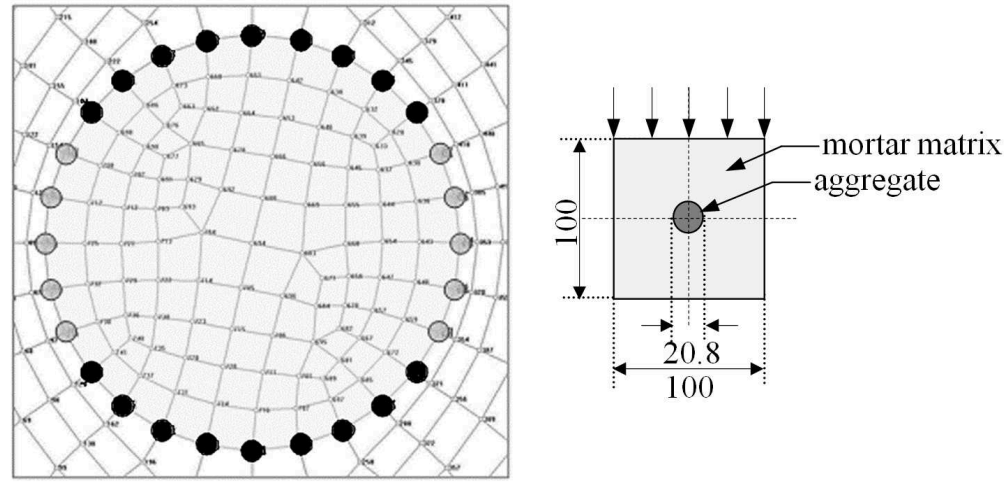

Fig. 2 Diagnostic of the ITZ responses

Additionally, the FEM program was run to compare the influence of the ITZ on the overall structural behavior. Fig. 3 shows the load-displacement responses for the structure with and without the presence of the ITZ. It was shown that for the same loading levels, a lower structural stiffness was obtained when the behavior of the ITZ was included. 


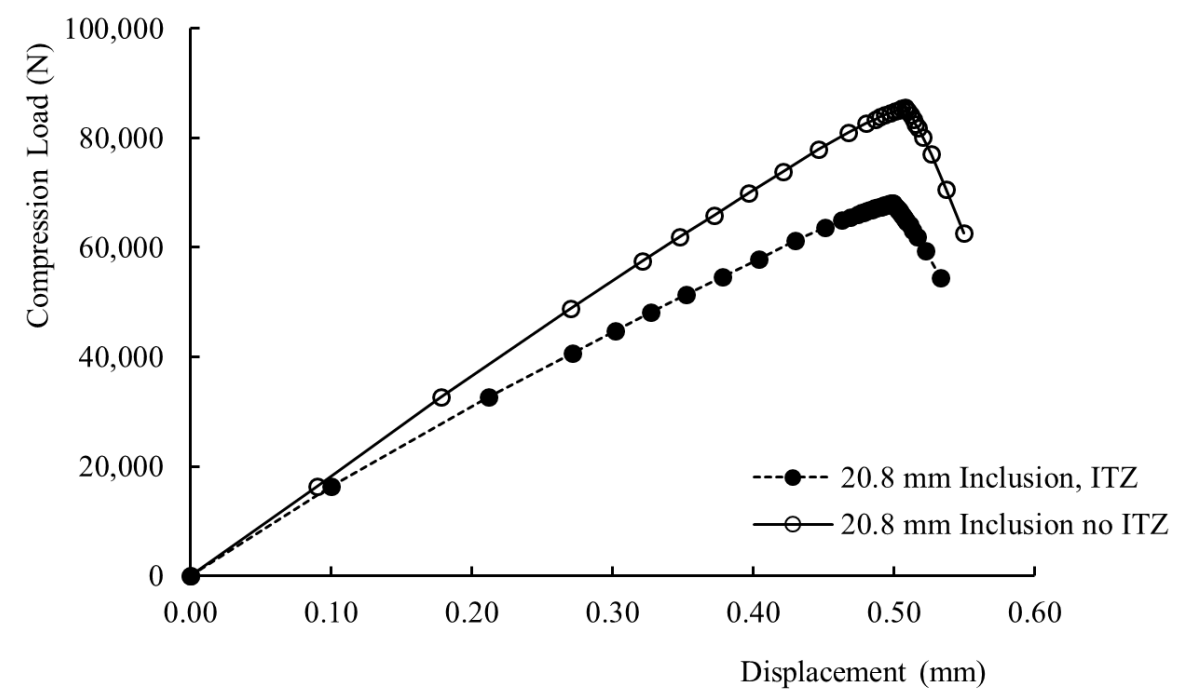

Fig. 3 ITZ response generated by the FEM

The program was further executed for a range of inclusion diameters; the load-displacement curves obtained by the FEM and the experimental specimens are presented in Fig. 4. The curves show that the FEM program produced a very close approximation to the real load-displacement behavior. The response was non-linear even at low loading levels. An ultimate was reached, and a post-peak response recorded. The behavior was brittle, and showed a similar structural stiffness response for all three inclusion diameters. The program tended to slightly underestimate the ultimate load of the laboratory specimens (Table 2).

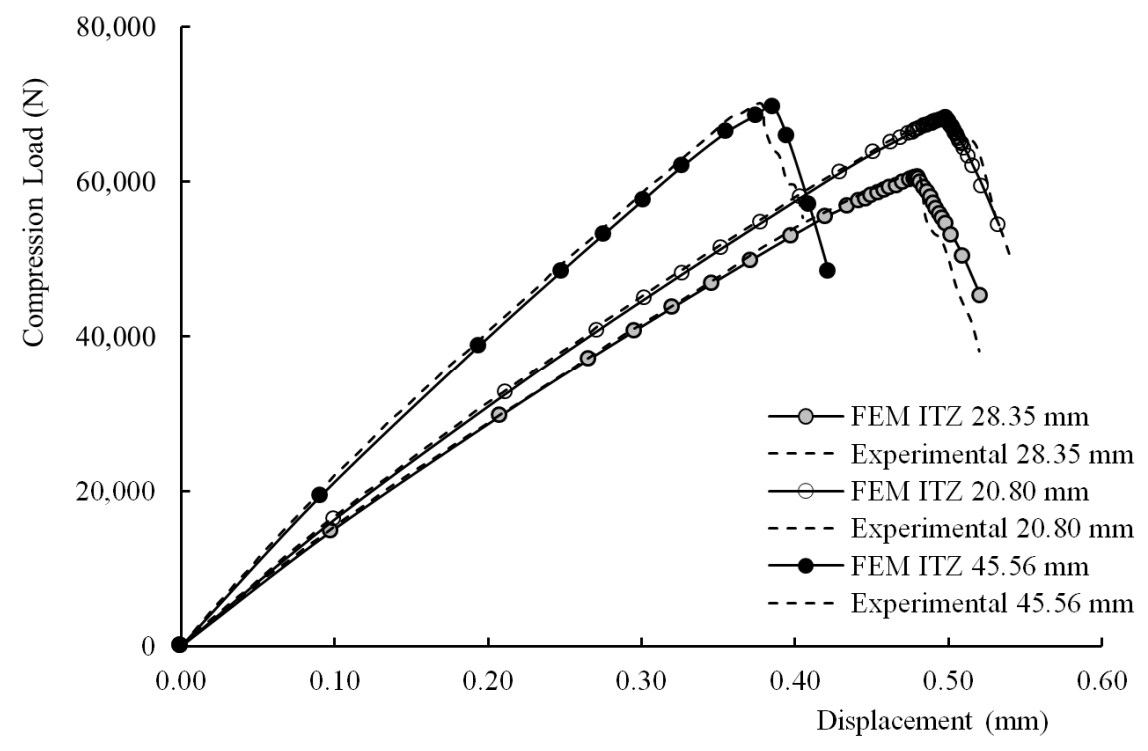

Fig. 4 Load-displacement response comparison

Table 2 Ultimate Load Comparison

\begin{tabular}{ccc}
\hline Inclusion & \multicolumn{2}{c}{ Ultimate Load $\mathrm{P}_{\text {ult }}(\mathrm{kN})$} \\
\cline { 2 - 3 } diameter $(\mathrm{mm})$ & experimental & FEM \\
\hline D20.80 & 68.60 & 68.09 \\
D28.35 & 61.27 & 60.54 \\
D45.56 & 70.09 & 69.67 \\
\hline
\end{tabular}

Conclusion and Future Research Work. It was clearly shown that the presence of the ITZ in the analysis of concrete could not be neglected. The developed FEM program was proven to produce a 
realistic and accurate prediction of the load-displacement response, including the ITZ. In addition, while the program correctly expressed the non-linear response under increasing monotonic loading, it also enabled the recording of post-peak behavior.

The modeling of the ITZ as a double spring was proven to be an excellent representation of the behavior of the interface. This model could basically be expanded for incorporation of all sorts of ITZs, since the normal and shear behavior are obtained through individual tests. However, further research needs to be conducted to investigate as whether the load-displacement relationship of the normal and shear stiffness is independent, as proposed in this research. The assumption that a normal spring in compression is equal to a totally bounded condition is also not entirely true.

To overcome these simplifications, more accurate and realistic laboratory test methods for the ITZ should be developed. The shear response as a function of the normal stress in compression should be studied. From here on, the stiffness matrix of the ITZ could be constructed reflecting the interaction between the shear and normal behavior. In the special case of bar reinforcement, the shear behavior can be approached by the constitutive model representing the bond between the bar and the concrete.

The FEM program was operated using the full square matrix based on the Gauss elimination method. When dealing with more complicated configurations, for example deformed steel bars that require very fine, complex meshing, the band-width method should be accessed, enabling a shorter running time.

\section{References}

[1] A.L. Han and P. Sabdono: Experimental Study to the Load-Displacement Response of the Interfacial Transition Zone in Concrete, $3^{\text {rd }}$ EACEF Conference, Yogyakarta, Indonesia (2011).

[2] S. Tudjono, A.L. Han and L.H. Hariwijaya: Reinforced Concrete Finite Element Analysis Incorporating Material Nonlinearity and Failure Criteria Aspects, Applied Mechanics and Materials, Vol. 284-287 (2012), pp. 1230.

[3] A.L. Han and Nuroji: The Normal and Shear Modulus Properties of the Interfacial Transition Zone in Concrete; Newly Developed Testing Procedures, $35^{\text {th }}$ Conference on Our World in Concrete and Structures, Singapore (2010).

[4] A.L. Han, S. Besari, Nuroji and C.T. Tam: Determination of the Interfacial Transition Zone Behavior between Aggregates and Mortar, in preparation.

[5] A.L. Han and J. Purnomo: Finite Element Modeling Incorporating Non-Linearity of Material behavior Based on the FIB Model Code 2010, Journal of Applied Technology and Innovations, Vol. 5 (2011), pp. 52.

[6] N.S. Ottosen: Constitutive Model for Short-Time Loading of Concrete, ASCE Journal of the Engineering Mechanics Division, Vol. 105 (1979), pp. 127.

[7] W.F. Chen: Plasticity in Reinforced Concrete, (Ross J. Publishing, 2007).

[8] W.F. Chen and A.F. Saleeb: Constitutive Equations for Engineering Material Vol. 1: Elasticity and Modeling, (John Wiley and Sons, 1982).

[9] F.J. Vecchio and M.P. Collins: The Modified Compression-Field Theory for Reinforced Concrete Elements Subjected to Shear, ACI Journal Proceedings, Vol. 83, no.2 (1986), pp. 219. 